\title{
L-Arginin in der Prophylaxe und Therapie von Diabetes mellitus
}

\author{
Horst Robenek, Burkhard Poeggeler
}

\section{Schlüsselwörter}

Altern, L-Arginin, Diabetes mellitus,

Stickstoffmonoxid (NO), asymmetrisches

Dimethylarginin (ADMA), Glukosestoffwechsel

\section{Zusammenfassung}

Die Nahrungsaminosäure L-Arginin und der daraus gebildete Botenstoff Stickstoffmonoxid (NO) spielen eine essenzielle Rolle bei der Erhaltung und Wiederherstellung der Gesundheit. Eine gezielte diätetische Zufuhr von L-Arginin kann nach neuesten Befunden Diabetes mellitus und anderen Stoffwechselstörungen vorbeugen und eine medikamentöse Therapie sinnvoll ergänzen.

Eine schnell alternde Gesellschaft braucht zunehmend gesundheitsfördernde Stoffe, die degenerative Prozesse und chronische Erkrankungen verhindern, umkehren oder zumindest vermindern können. Dazu bieten sich ganz besonders gut verträgliche Naturwirkstoffe an. Hier entwickeln sich vielfältige Perspektiven für eine regenerative Biomedizin der Zukunft [1]. Das Konzept, die molekularen Mechanismen und Mediatoren der Gesundheitsförderung zu analysieren und zu erforschen, wird ein besseres Verständnis für deren physiologische Basis und Dynamik ermöglichen. Die naturwissenschaftlichen Grundlagen der biomedizinischen Forschungen auf diesem Gebiet bilden damit ein wichtiges Fundament für vielversprechende neue Ansätze, welche die Versorgung in Prävention, Therapie und Rehabilitation entscheidend verbessern können [2].

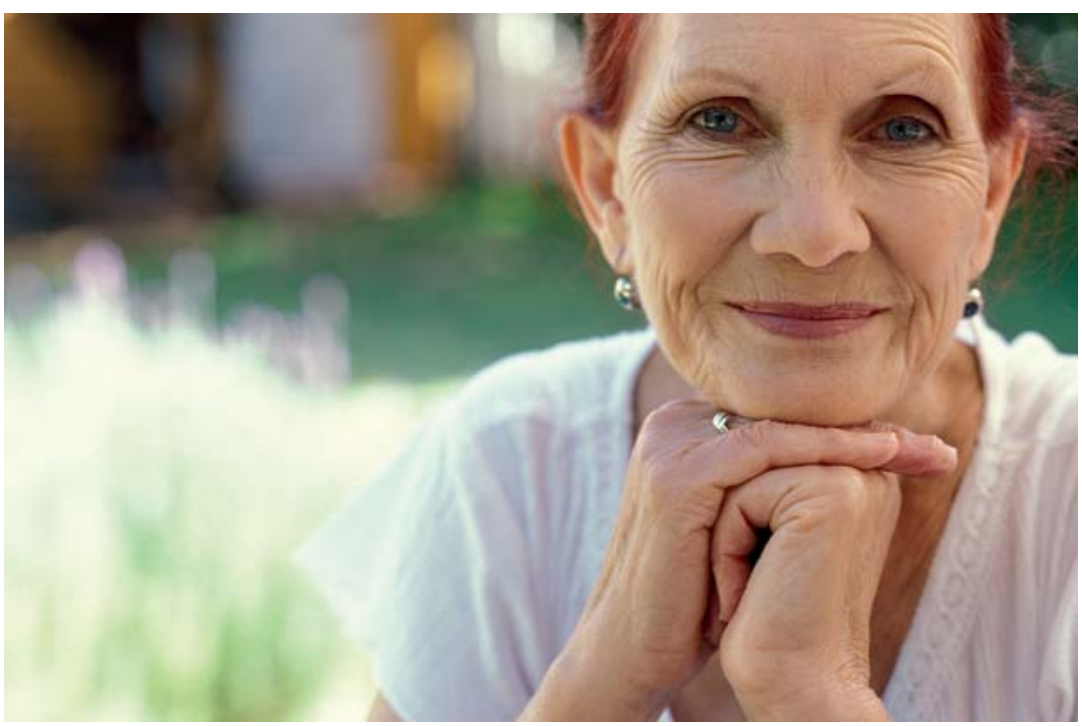

Besonders im Alter kommt es auf eine ausgewogene Ernährung an. L-Arginin in Lebensmitteln oder als Nahrungsergänzung kann altersbedingten Störungen des Glukosestoffwechsels entgegenwirken. (c) Jupiterimages; nachgestellte Situation

Die Prävalenz von Diabetes mellitus steigt im Alter stark an [3]. Aktuelle Studien belegen, dass etwa jede dritte Frau und jeder vierte Mann im Alter von 75 bis 80 Jahren in Deutschland an Diabetes mellitus erkrankt. Diese älteren Menschen leiden oft auch an anderen Stoffwechselstörungen. Daher sind gerade auf diesem Gebiet neue Ansätze gefragt.

\section{L-Arginin als mitogene und mitotrophe Aminosäure}

Die Nahrungsaminosäure L-Arginin ist für die antioxidative Protektion und bioenergetische Stimulation von entscheidender Bedeutung [2]. Es konnte nachgewiesen werden, dass L-Arginin die Stoffwechseleffizienz erhöht und damit die Gesundheit auch im fortgeschrittenen Alter erhalten kann [2]. Eine L-Arginin-reiche Ernährung oder eine Supplementierung mit L-Arginin kann über eine vermehrte Bildung des protektiven Botenstoffs Stickstoffmonoxid (NO) die Zuckerstoffwechseleffizienz verbessern und damit altersbedingten Stoffwechselstörungen entgegenwirken [4-6].

Asymmetrisches Dimethylarginin (ADMA) und andere endogene methylierte Argininmetabolite fungieren als Gegenspieler von Arginin [2,6-9] und können daher Prozesse auslösen, die zu gefährlichen Stoffwechselstörungen führen. Die Konzentrationen von ADMA und methylierten Argininmetaboliten sind bei fast allen Personen mit Stoffwechselproblemen wie Diabetes mellitus stark erhöht $[2,6]$. Sie verringern 
Tab. 1 Studie nach Poeggeler et al. [2].

\begin{tabular}{lcc}
\hline & Placebo & L-Arginin $(\mathbf{p}<\mathbf{0 , 0 5})$ \\
\hline ADMA $(\mu \mathrm{mol} / \mathrm{l})$ & $4,78 \pm 0,38$ & $2,23 \pm 0,21$ \\
Homocystein $(\mu \mathrm{mol} / \mathrm{l})$ & $18,57 \pm 1,41$ & $11,05 \pm 1,04$ \\
Superoxidanionradikale (nmol/min/mg Protein) & $0,41 \pm 0,04$ & $0,18 \pm 0,03$ \\
Peroxide $(\mathrm{nmol} / \mathrm{min} / \mathrm{mg}$ Protein) & $0,38 \pm 0,05$ & $0,16 \pm 0,02$ \\
Blutzucker $(\mathrm{mg} / \mathrm{dl})$ & $152 \pm 18$ & $118 \pm 14$ \\
$\mathrm{HbA}_{1 \mathrm{c}}(\%)$ & $7,5 \pm 0,3$ & $6,4 \pm 0,5$ \\
\hline
\end{tabular}

die Bioverfügbarkeit von L-Arginin und hemmen die Bildung von NO bei gleichzeitig erhöhtem Verbrauch dieses Radikalfängers [2,6]. Erhöhte ADMA-Spiegel steigern den Bedarf an L-Arginin daher erheblich, vermindern kompetitiv die argininabhängige NO-Synthese und verursachen einen sehr gefährlichen altersbedingten NO-Mangel [2,6], der immer mit einem erhöhten nitrosativen Stress unter Bildung hochreaktiver und zerstörerischer Radikale verbunden ist.

\section{Stoffwechselstörungen natürlich behandeln}

Ein aktives, gesundes Endothel bildet ausreichend NO aus dem reichlich vorhandenen L-Arginin und kann so die Insulinsensitivität [7] und die Glukoseverwertung verbessern $[2,6,7]$, während ADMA und die anderen Argininantagonisten die für den Diabetes typischen Stoffwechselstörungen verstärken $[2,6$, 7]. Diabetes mellitus ist ebenso wie das Altern selbst immer mit einem absoluten und relativen Mangel an L-Arginin verbunden $[2,8]$. Sowohl im Alter als auch bei Diabetes mellitus kommt es daher zu einer verminderten Bildung von NO und damit zu den entsprechenden Stoffwechselstörungen [2,6-8]. Der mit dem NO-Mangel verbundene nitrosative Stress führt zu altersbedingten degenerativen Prozessen und oxidativen Schäden mit einer stark verminderten Stoffwechseleffizienz und einer gestörten Glukoseverwertung. Gesund zu Altern bedarf daher einer vermehrten $\mathrm{Zu}-$ fuhr von L-Arginin. Da allein die Konzentrationen des Argininantagonisten
ADMA schon bei gesunden älteren Menschen um etwa das 4 -fache ansteigen [2, 9], benötigt man eine etwa 40-fach erhöhte L-Arginin-Versorgung.

L-Arginin wirkt den schädlichen Auswirkungen von ADMA entgegen [2, 9]. Die gegenüber jungen Erwachsenen im fortgeschrittenen Alter 4-fach erhöhten ADMA-Konzentrationen können durch L-Arginin signifikant reduziert werden. L-Arginin senkt auch die altersbedingt erhöhten Konzentrationen an Superoxidanionradikalen und Peroxiden. Auch das den ADMA-Abbau hemmende Homocystein wird verringert. Diese antioxidative Protektion führt zu einer Senkung der Blutzucker- und der $\mathrm{HbA}_{1 \mathrm{c}}$-Werte auch im hohen Alter $(\rightarrow$ Tab. 1).

Ganz ähnliche Befunde konnten bereits 2008 in einer großen multizentrischen Anwendungsbeobachtung ${ }^{1}$ demonstriert werden [10]. Diese Ergebnisse erscheinen vor dem Hintergrund der anderen neuen großen Studien zu L-Arginin und Diabetes mellitus [4-6] aussichtsreich und vielversprechend. L-Arginin ist essenziell für die Gefäßgesundheit [11]. Die therapeutische Anwendung der Aminosäure im Rahmen der Durchblutungsförderung und antioxidativen Protektion bei Diabetes mellitus ist gerade auch im hohen Alter zudem seit langem erfolgreich etabliert [12]. Die laufenden großen Anwendungsbeobachtungen und klinisch kontrollierten Studien können Aufschluss über den Nutzen von L-Arginin in der Vorbeugung und Behandlung von diabetischen Stoffwechselstörungen geben.

${ }^{1}$ Prüfpräparat Telcor ${ }^{\circledR}$ Arginin plus
Ziel sollte es sein, den Risikofaktor Diabetes mellitus für den betagten Menschen so gering wie möglich zu halten und dabei eine optimale Gesamttherapie dieser Stoffwechselprobleme einzuleiten, die die $\mathrm{HbA}_{1 \mathrm{c}}$-Werte dauerhaft unter $7,5 \%$ halten $[1-3,6]$. Auch in der geriatrischen Population sollte der Blutzuckerspiegel $150 \mathrm{mg} / \mathrm{dl}$ nicht überschreiten [3]. Nur bei einem Nüchternblutzucker, der $126 \mathrm{mg} / \mathrm{dl}$ dauerhaft nicht übersteigt, ist eine zusätzliche medikamentöse antidiabetische Therapie nicht notwendig [3]. Eine antidiabetische Therapie kann sehr wirkungsvoll auf natürliche und physiologische Weise optimiert werden. Dabei spielen eine abwechslungsreiche und ausgewogene Ernährung sowie vermehrte Bewegung und gezielte Entspannung eine Schlüsselrolle [3,6]. Eine ausreichende Nährstoffversorgung oder Supplementierung mit L-Arginin kann als Ergänzung und Unterstützung der medikamentösen Therapie positive Wirkungen auf die dem Diabetes mellitus zugrunde liegenden Stoffwechselstörungen entfalten und sollte daher so früh wie möglich zur adjuvanten Therapie dieser vor allem im fortgeschrittenen Alter auftretenden Probleme eingesetzt werden.

\section{Fazit}

Die Nahrungsaminosäure L-Arginin kann altersbedingten Störungen des Glukosestoffwechsels entgegenwirken. L-Arginin verbessert die Glukoseverwertung und verhindert die gesundheitlichen Schäden, die bei erhöhtem Blutzuckerspiegel auftreten. Eine L-Arginin-reiche Ernährung oder die diätetische Zufuhr der für die Gesundheit essenziellen Aminosäure L-Arginin erscheint daher vor allem im Alter empfehlenswert.

Interessenkonflikte: Prof. Dr. Horst Robenek hat keine Interessenkonflikte. PD Dr. Burkhard Poeggeler arbeitet auch für QUIRIS Healthcare, Anbieter von diätetischen Argininprodukten in Deutsch- 
land. Diese wirtschaftliche Verbindung wird deshalb hier ausdrücklich angezeigt.

\section{Literatur}

[1] Poeggeler B. Umkehrung des Alterungsprozesses durch mitochondriale Nährstoffe? 2012. Im Internet: www.symptome.ch/ blog/umkehrung-des-alterungsprozessesdurch-mitochondriale-nahrstoffe-2/. Stand: Januar 2014

[2] Poeggeler B, Weinberg J, Pappolla M. Arginin und ADMA: Gesundheit im Alter. Abstractband 37. Wissenschaftlicher Kongress der Deutschen Hochdruckliga e. V. DHL ${ }^{\circledR}$ - Deutschen Gesellschaft für Hypertonie und Prävention in Münster, 12.14. Dezember 2013; FV-20: 23-24. Im Internet: http://hypertonie2013.rh-kunde.de/ files/2013/03/Abstractband_Hypertonie.pdf

[3] Jungmayr P. Ziele setzen, Mangelernährung vermeiden. Deutsche Apotheker Zeitung 2013; 50: 34-35

[4] Monti LD, Casiraghi MC, Setola E. L-arginine enriched biscuits improve endothelial function and glucose metabolism: a pilot study in healthy subjects and a cross-over study in subjects with impaired glucose tolerance and metabolic syndrome. Metabolism 2013; 62(2): 255-264

[5] Monti LD, Setola E, Lucotti PC et al. Effect of a long-term oral l-arginine supplementation on glucose metabolism: a randomized, double-blind, placebo-controlled trial. Diabetes Obes Metab 2012, 14(10): 893-900

[6] Bøtker HE, Møller N: ON NO - the continuing story of nitric oxide, diabetes, and cardiovascular disease. Diabetes 2013; 62(8): 2645-2647

[7] Siervo M, Bluck LJ: In vivo nitric oxide synthesis, insulin sensitivity, and asymmetric dimethylarginine in obese subjects without and with metabolic syndrome. Metabolism 2012; 61(5): 680-688

[8] Ramirez-Zamora S, Méndez-Rodriguez ML, Olguin-Martinez M. Increased erythrocytes by-products of arginine catabolism are associated with hyperglycemia and could be involved in pathogenesis of type 2 diabetes mellitus. PLoS ONE 2013; 8(6): e66823

[9] Bode-Böger SM, Muke J, Surdacki A. Oral $\mathrm{L}$-arginine improves endothelial function in healthy individuals older than 70 years. Vasc Med 2003; 8: 77-81

[10] Jung K, Petrowicz O. L-Arginin und Folsäure bei Arteriosklerose. Ergebnisse einer prospektiven, multizentrischen Verzehrsstudie. Perfusion 2008; 21: 148-156

[11] Robenek H, Poeggeler B. L-Arginin: essenziell für die Gefäßgesundheit. OM - Zs $f$ Orthomol Med 2013; 3: 16-19

[12] Robenek H, Poeggeler B. Therapeutische Anwendung von L-Arginin. OM - Zs f Orthomol Med 2013; 3: 21-23

online: http://dx.doi.org/10.1055/s-0033-1360399

Erratum zu

Claus Schulte-Uebbing et al.: Hashimoto-Thyreoiditis. OM 2013; 4: 20-24 Im Artikel auf S. 20 hat sich ein Fehler eingeschlichen. In der Bildunterschrift muss es richtig heißen:

„Die Hashimoto-Thyreoditis geht mit einer Verkleinerung der Schilddrüse einher.“

Autor und Verlag bitten diesen Fehler zu entschuldigen.

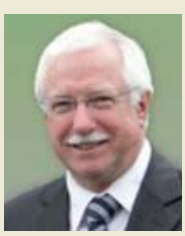

Prof. Dr. Horst Robenek Universitätsklinikum Münster Albert-SchweitzerCampus 1 Domagkstraße 3 48149 Münster E-Mail: robenek@ uni-muenster.de

Prof. Dr. Horst Robenek war von 1987 bis 1989 Heisenberg-Professor der Deutschen Forschungsgemeinschaft. 1989 wurde er als Universitäts-Professor an das Leibniz-Institut für Arterioskleroseforschung berufen und wurde zum Leiter des Departments für Zellbiologie und Ultrastrukturforschung bestellt. Von 1994 bis 1999 war Prof. Robenek Präsident der Deutschen Gesellschaft für Zellbiologie, 2007 wurde er zum Direktor des Leibniz-Instituts für Arterioskleroseforschung ernannt. Seit seiner Emeritierung Anfang 2012 ist er Senior-Professor an der Medizinischen Fakultät der Westfälischen Wilhelms-Universität in Münster. Seine Forschungsschwerpunkte sind die intrazellulären Vorgänge des Fettstoffwechsels unter besonderer Berücksichtigung der Speicherung von Fetten in vaskulären Zellen bei der Arteriosklerose.

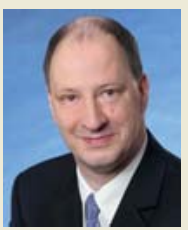

PD Dr. Burkhard Poeggeler Eisenacher Straße 7 37085 Göttingen E-Mail: bpoegge@ gwdg.de

PD Dr. Burkhard Poeggeler wurde 1998 an die University of South Alabama in Mobile/ Alabama zum Professor für Pathologie berufen und zum Direktor des Instituts für molekulare Neuropathologie am assoziierten Mobile Medical Hospital Center ernannt. Er habilitierte 2006 an der Georg-August-Universität Göttingen und arbeitet seitdem als Privatdozent am Goettingen Research Campus und als Hochschullehrer an der Fakultät für Biologie und Psychologie der Universität Göttingen. Dr. Poeggeler entdeckte die Elektronenübertragung als entscheidenden Mechanismus der Radikalentgiftung und hat das SI-System zur Radikaldetoxifikation mitbegründet. 